\title{
Design and Realization of Modularized Marine Observation Data Transmission System
}

\author{
Yubo Wen \\ South China Sea Information Center of State Oceanic Administration, Guangzhou 510310, China \\ wenyubo998@163.com
}

Keywords: Data transmission, modularized, transmission system, C/S architecture.

\begin{abstract}
Observation expansion has brought new requirements for validity, security, stability and integrity of observation data transmission in recent years. Under this background, this paper designs a modularized marine observation data transmission system, describes its overall architecture and software design and proposes level-free data transmission system for overall control of data transmission. In the end, the environment for marine observation data transmission is simulated to test the system, proving that the proposed system can improve the efficiency of data transmission and realize the unified transmission, monitoring and management of data.
\end{abstract}

\section{Introduction}

Marine observation data transmission, as a fundamental task of the whole marine observation system, encompasses the collection, transmission, storage, distribution and application of observation data, making it the basic facility for marine observation \& forecast and continuous disaster prevention and relief in sea area. Now, China has preliminarily established a three-dimensional marine environmental observation and transmission system consisting of shore-based monitoring station, buoy, voluntary ship, ocean satellite, and ground transmission network, which plays a significant role in the fields of marine observation \& forecast and disaster prevention and relief.

Continuous development of observation \& forecast and disaster prevention and relief in sea area has increased the dependence on the support from basic information year by year, and demands the inclusive, continuous and normalized support from basic information to marine activities by means of marine observation data transmission system. The existing marine observation data transmission system gradually reveals a lot of shortcomings, e.g. low transmission efficiency, poor security and unfavorable scalability, which obstructs the sustainable development of marine activities to some extent.

To solve the problems of the existing marine observation data transmission system, this paper designs a modularized marine observation data transmission system and intends to make a stable, fast, secure and controllable marine observation data transmission system from the perspectives of basic transmission network, data transmission software and security facility construction.

\section{System Design}

\subsection{Design of Overall Architecture}

The overall architecture of software is presented in Fig. 1. Marine observation data transmission software is designed based on the $\mathrm{C} / \mathrm{S}$ architecture and utilizes the modular design to convert each function into an independent module. The modules can be called by means of loose coupling to keep their independence, and an reserved interface for module development is provided to facilitate the expansion of functions in the future. At the module configuration level, the combination of modules is user-defined to form different business subsystems, which satisfy the needs of different businesses and service objects, and guarantee the flexibility of system and the adaptability to change of needs to the maximum. The overall architecture of software is divided into six levels: 
Network hardware equipment level. It provides the fundamental network hardware support for operation of software system, including network service, host service, storage service and backup service.

Data storage level. The system combines Sql Server database, Access database and file storage for data storage. Among them, Sql Server is classified into relational database and spatial database. Relational database is used to store the data generated in the system, e.g. observation data, monitoring data and log data, etc., while spatial database applies to the GIS map demonstration and operation of the system. Access is a desktop database for storing the local data generated by the system, e.g. transmission logs and login information, etc. File storage is suitable for storing the received original and backup files on the local disk.

Data access level. To improve the security of database system, middleware technology is employed to realize the database access and management between application system and database.

Business logic level. Based on business needs and modular design principles, the system functions are allocated to six major modules, i.e. data transmission, data management, network management, observation equipment management, transmission strategy management, and system management. Additionally, it is planned to reserve the interface for module development for functional scalability, in order to satisfy the varying needs.

Module configuration level. The user-defined combination of modules results in different business subsystems to satisfy the needs of different businesses and service objects.

System application level. Different combinations of modules are encapsulated into independent subsystems to serve their users.

\subsection{Design of Data Transmission System}

The existing marine observation data transmission system employs the top-down hierarchical transmission system, which consists of four levels, e.g. ocean station, central station, sea area and national administration. Each level encompasses multiple transmission nodes, and each node submits the data level by level or across level through the preset IP, as shown in Fig. 1.

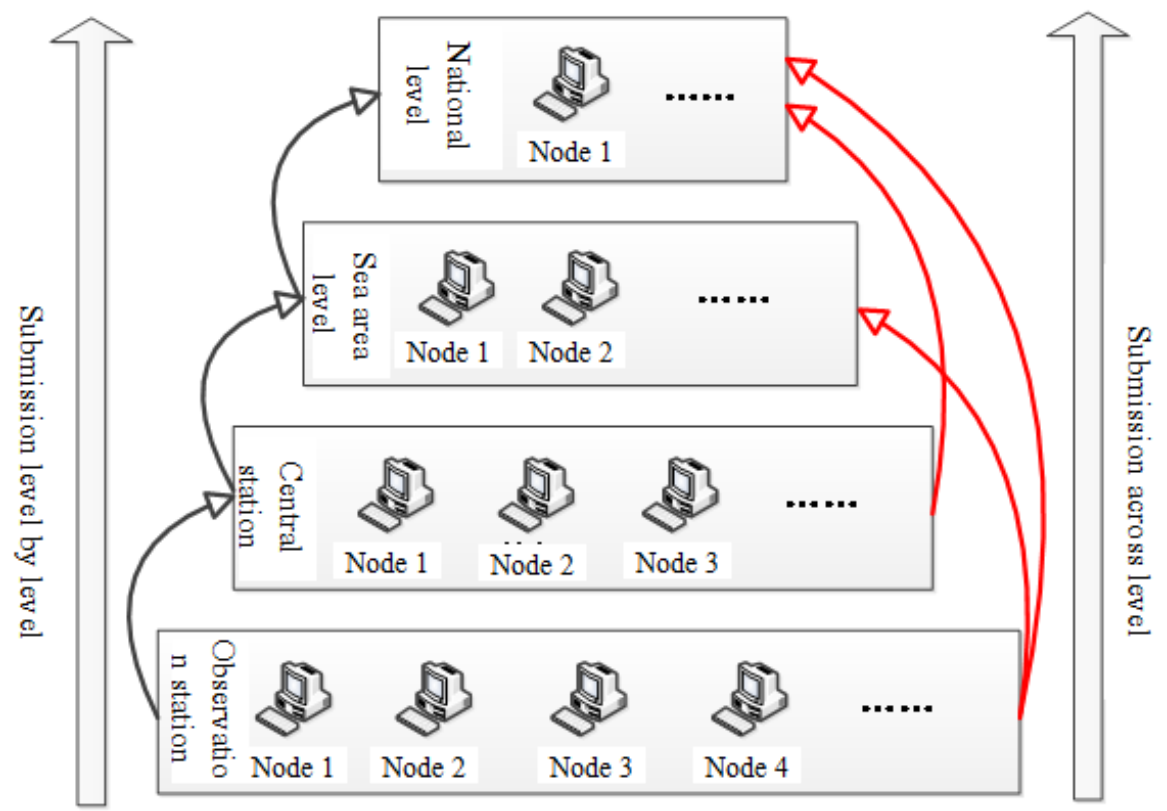

Fig. 1 Leveled Data Transmission Model

This transmission model has the following shortcomings:

(1) In terms of management, it lacks the overall control mechanism, so it is impossible to realize the unified control of all nodes.

(2) In terms of data transmission, lack of management and control mechanism may cause the whole transmission link out of control since each node can control the object of data sending independently.

(3) Scalability and adaptability of software architecture are poor due to its inflexible transmission levels. If any change happens to the levels of organizational architecture, but the existing 
transmission system is unable to adjust to such change correspondingly, the system will have to be redeveloped in the end.

To solve the above problems, better satisfy the needs of current business and cope with the risk caused by the variation of needs in the future, it is planned to establish a flattened data transmission model, weaken the hierarchical relations and change the 4-level hierarchical structure. Hence, a level-free data transmission system (see Fig. 3) is employed to provide no level or sequential relation for all nodes. In the system, the global data transmission strategy is configured through back-end service, and each node delivers the data to other nodes based on the preset strategy, which allows two-way and concurrent transmission.

The level-free data transmission model has the following advantages:

(1) Controllability of transmission link

Each node must connect to the back-end service side and learn the associated data transmission strategy prior to initiation of data transmission, and implement the data transmission strictly based on the strategy, so as to help monitor and learn about the status of its data transmission and realize the unified management and control of the whole transmission system.

(2) Adaptability to change of needs

By configuring the strategy at the service side, the direction of data transmission at each node can be controlled. When any change happens to the organizational architecture of transmission system and the needs of transmission, the strategy of some nodes can be modified to satisfy new needs without redevelopment of software system. Hence, the level-free transmission model can better adapt to the risk caused by change of needs.

(3) Flexibility of node management

When it is necessary to add or delete a node, the connection with service side must achieved first. After it is reviewed and authorized by service side, the node can be added to or deleted from the transmission system, which make node management significantly convenient and flexible.

(4) Security of data transmission

Service side is responsible for unified control of data transmission, and nodes are not allowed to set the transmission goal independently, which prevent artificial disturbance and guarantee the security of data transmission.

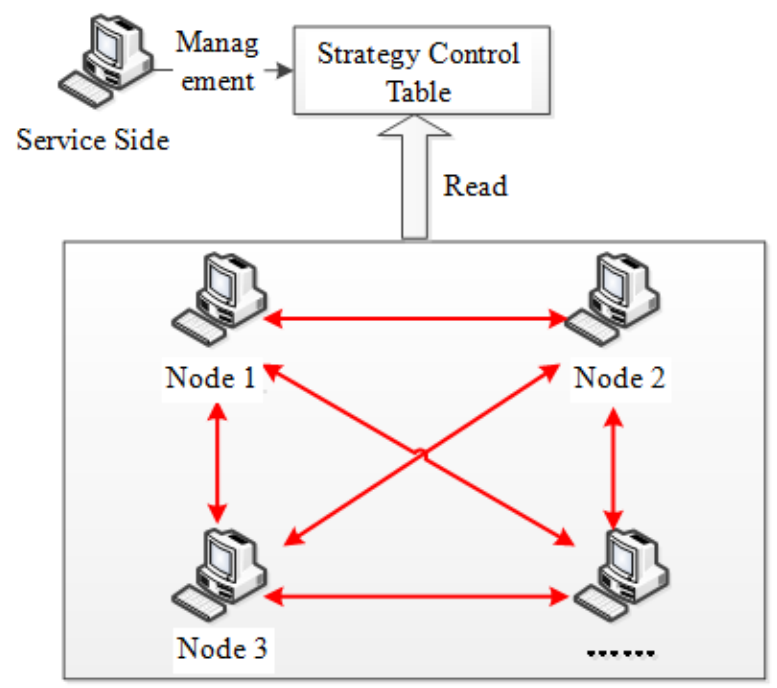

Fig. 2 Level-free Data Transmission System

\section{Main Functions of the System}

\subsection{Observation Data Transmission}

(1) Data selection: Select the local file to be sent, and support the selection of multiple files and folder. 
(2) Data sending: Obtain the corresponding authority and transmission strategy from back-end system server prior to sending, configure based on the strategy, and send the data through FTP protocol.

(3) Data storage: Store the collected data of stations in the server automatically according to the specified regulations and standards, and allow to choose the destination of local storage.

\subsection{Observation Data Management}

(1) Quality control: Realize the quality control of data by analyzing the integrity, reasonableness and consistency of data to guarantee the authenticity and accuracy of warehoused data.

(2) Data warehousing: Process the marine observation data in a standardized manner to comply with the unified regulations and standards, and employ the unified codes and formats to download and warehouse the data.

(3) Data inquiry: Inquire about the data observed at each monitoring point, e.g. voluntary ship, station and buoy, in a real-time manner, including every hour, half an hour, or minute. The time period of inquiry is optional, and the types of data available include voltage, instantaneous wind speed, instantaneous wind direction, wind speed, wind direction, atmospheric pressure, temperature, humidity, water temperature, salinity, wave height, wave direction, wave period, rainfall, tide level, flow velocity, flow direction, $\mathrm{CO} 2$ and water quality, etc. The inquired data can be exported to local (subject to strict authority control), and the historic data can be converted into curves to vividly reflect the change trend of data.

(4) Data monitoring. Realize the monitoring of observation data, and monitor the real-time data observed at each monitoring point in a real-time manner. The GIS map is employed to vividly display the locations of buoys, voluntary ships, stations, radars and emergency vehicles, and show the detailed monitoring data of each monitoring station in a real-time manner. It can vividly and clearly demonstrate the information of each monitoring point, and monitor the status of transmission system in a real-time manner. On the other hand, it monitors the status of transmission system and displays the status of data transmission between stations and the status of data download and data upload at the current station.

\subsection{Data Transmission Strategy Management}

(1) Registration management: Registration must be conducted before using for the first time. Operation may be conducted after passing the back-end review.

(2) Node management: Review the application for access to front-end system, add or delete a node, modify the parameters of node, and allocate the authority to nodes, etc.

(3) Data transmission strategy management: By authority setting of service side, control the flow direction of data transmission in the front-end system, i.e. setting the object of sending for each node.

\subsection{Database Design}

Considering the storage of relational data and spatial data for the whole system, the databases constructed should have the following functions:

(1) Support for the storage of spatial data;

(2) Quick data search;

(3) Ability to manage mass data;

(4) Stability and security of system;

(5) Client's support for Windows, including database interface;

(6) Compatibility with operating system.

Meanwhile, Sql server database is taken as the primary management software for back-end database of the system considering the convenience of database maintenance due to its favorable security, stability and scalability. On the other hand, the system generates massive local data (e.g. parameter information, log information, and file transmission information, etc.) after operations, so it is necessary to install the Access desktop database at each front-end client for convenient storage of these data and operation \& maintenance of the system.

The databases employed in data transmission software mainly include geographic database, information database, management database, system database and Access desktop database.

The overall structure of system databases is as follows: 


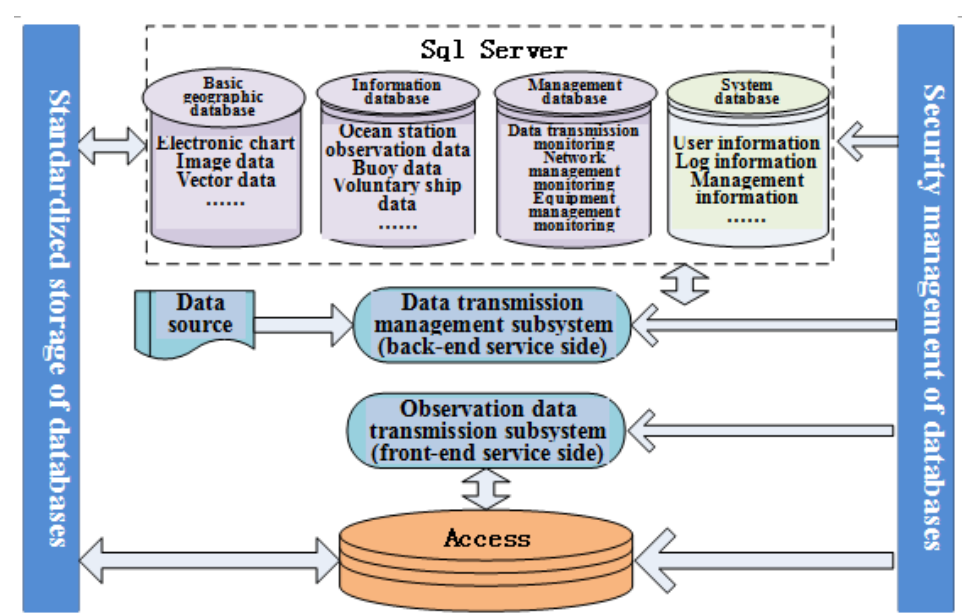

Fig. 3 Overall Structure of System Databases

All these databases must be kept in the unified data center during storage and management. Mature data warehouse technology and advanced interoperation technology are utilized to process the massive, polygenetic and heterogeneous marine information, and integrate mass data into the unified database environment for effective management in terms of theme, region and time. Additionally, the data access interfaces of different levels and the tools for multidimensional and dynamic expression and analysis are provided to overcome the defects including complicated and disorderly data, few application data in massive original data, and little knowledge from diverse data, in order to provide the polygenetic and multi-scale data support for marine management.

\section{Conclusion}

Marine observation data transmission software is crucial to the construction of marine observation system. Along with the continuous development of observation in recent years, stable and efficient data transmission plays a significant role in supporting the forecast and alarm, disaster prevention and relief of marine observation effectively. This paper designs a modularized marine observation data transmission system, and proposes a level-free data transmission system for overall control of data transmission. In the end, this paper simulates the environment for marine observation data transmission for testing the system, and the results prove that the proposed system can improve the efficiency of data transmission and realize the unified transmission, monitoring and management of data.

\section{References}

[1] Zhang Jiantao, Han Jiaxin. Research on standardization technology of data transmission of marine observation platforms. Ocean Technology, 2011, 30(2): 41-45.

[2] Wei Fangzhong, Wen Xiangcai, Li Gang et al. Design concept of national-level data transmission system for water environmental monitoring. Environmental Monitoring in China, 2011, 27(1): 38-41.

[3] Zhang Jun, Tao Linmi, Yang Chenke et al. Heterogeneous data transfer software platform for on-the-spot archiving system. Computer Applications, 2006, 26(10): 2486-2489.

[4] Huang Shiquan. FTP protocol analysis and security research. Microcomputer Information, 2008, 24(6): 93-95.

[5] Ji Peng, Zhang Chenghui, Sun Rupeng. The system of the real time marine data transmission network based on XML . Marine Forecasts, 2006, 23(2): 45-51.

[6] Mei Sai, Gao Jinyao, Zhao Tiehu et al. A bathymetry data management system based on Client/Server Mode [J]. Marine Science Bulletin, 2011, 30(4): 361-366. 